\title{
Comparing telehealth-based and clinic-based group cognitive behavioral therapy for adults with depression and anxiety: a pilot study
}

This article was published in the following Dove Press journal:

Clinical Interventions in Aging

7 May 2014

Number of times this article has been viewed

\author{
Nasreen Khatri \\ Elsa Marziali \\ Illia Tchernikov \\ Nancy Shepherd \\ Rotman Research Institute, \\ Toronto, ON, Canada
}

Correspondence: Nasreen Khatri Rotman Research Institute, Baycrest, Room 714, 3560 Bathurst Street, Toronto, ON, Canada M6A 2EI

Tel + I 4167852500 extension 3515 Fax + I 4167852862

Email nkhatri@research.baycrest.org
Background: The primary objective of this pilot study was to demonstrate reliable adherence to a group cognitive behavioral (CBT) therapy protocol when delivered using on-line video conferencing as compared with face-to-face delivery of group CBT. A secondary aim was to show comparability of changes in subject depression inventory scores between on-line and face-to-face delivery of group CBT.

Methods: We screened 31 individuals, 18 of whom met the criteria for a DSM-IV (Diagnostic and Statistical Manual of Mental Disorders, 4th Edition) diagnosis of mood and/or anxiety disorder. All qualifying participants had the necessary equipment (computer, webcam, Internet) for participation in the study, but could exercise their preference for either the on-line or face-to-face format. Eighteen completed the 13 weekly session intervention program (ten face-to-face; eight video conferencing). We coded adherence to protocol in both intervention formats and generated pre-post changes in scores on the Beck Depression Inventory Second Edition (BDI-II) for each participant.

Results: Application of the CBT protocol coding system showed reliable adherence to the group CBT intervention protocol in both delivery formats. Similarly, qualitative analysis of the themes in group discussion indicated that both groups addressed similar issues. Pre-post intervention scores for the BDI-II were comparable across the two delivery formats, with $60 \%$ of participants in each group showing a positive change in BDI-II severity classification (eg, from moderate to low symptoms).

Conclusion: This pilot study demonstrates that group CBT could be delivered in a technology-supported environment (on-line video conferencing) and can meet the same professional practice standards and outcomes as face-to-face delivery of the intervention program.

Keywords: psychotherapy, gerontology, mood disorders, computer-based, cognitive behavioral therapy

\section{Introduction}

The primary objective of this pilot study was to demonstrate reliable adherence to a group cognitive behavioral (CBT) therapy protocol when delivered using on-line video conferencing as compared with face-to-face delivery of group CBT. A secondary aim was to show comparability of changes in depression inventory scores between on-line and face-to-face delivery of group CBT. There is an increasing need to improve access to mental health services for patients with clinical depression, which is the most prevalent psychiatric disorder worldwide. ${ }^{1}$ Adults in their 50s and 60s are particularly vulnerable to clinical depression and anxiety due to high rates of cooccurring obesity, cardiovascular disease, and type 2 diabetes. ${ }^{2-4}$

The prevalence of depression escalates in tandem with the increase in incidence of chronic disease as people age. In North America, approximately $80 \%$ of adults over 
the age of 60 years have a chronic disease, and of these, $30 \%$ have three or more chronic conditions..$^{5}$ A major concern is that having a chronic disease exacerbated by the presence of depression and/or anxiety results in poor adherence to prescribed treatment and consequent deterioration in health and overall quality of life. $\left(\right.$ Katon $\left.^{6}\right)$.

\section{Background}

CBT is an evidence-based, time-limited collaborative form of psychotherapy delivered individually or in a group format. ${ }^{7}$ Group CBT for depression has been shown to be effective for adults across the age spectrum, decreasing symptom levels (eg, low mood, sleep difficulties) and improving cognitive function. ${ }^{8-11} \mathrm{CBT}$ is particularly appropriate for treating depression and anxiety in older adults with complex medical profiles, and can be used when antidepressant drugs are contraindicated or have intolerable side effects. Group CBT is an efficacious and cost-effective way of treating depression in an aging population that is undertreated despite the increasing prevalence of mood disorders in this age group. ${ }^{12}$

In addition, studies have shown consistently that CBT is more effective than medication alone for reducing the risk of relapse, resulting in treatment efficacy and overall costeffectiveness. ${ }^{2}$ A group format for providing mental health services to adults helps to prevent social isolation, which is a risk factor for poorer mental health in adults with limited support systems. ${ }^{13}$

Technology is being used increasingly to provide various forms of psychotherapy and counseling. For example, telephones, video telephones, and Internet web applications are used to support interactions between therapist and client/patient. CBT has been provided via the Internet using email exchange between patient and therapist in addition to providing web-based information about the management of depression. ${ }^{14}$ While technology has been used to provide individual CBT, we found no studies of group CBT delivered using Internet-based video conferencing, nor any studies that compared process and outcomes of video conferencing group CBT with those of face-to-face group CBT. The aim of this study was to demonstrate that a technology-supported form of group CBT would meet the same clinical practice standards and achieve outcomes similar to those achieved using clinic-based group CBT.

\section{Materials and methods}

The study received approval from the research ethics board of Baycrest, an academic health sciences center in
Toronto, Canada. The study was implemented in a clinic treating adult mood and anxiety disorders. We screened 31 potential participants aged in their 40 s to 70 s. Participants were referred by their physicians. Potential participants who wished to participate in the study were contacted, informed of the study, and invited to attend a DSM-IV (Diagnostic and Statistical Manual of Mental Disorders, Fourth Edition)-SCID (Structured Clinical Interview for DSM-IV Axis I Disorders) assessment interview and to complete a baseline study questionnaire. All subjects provided their written informed consent.

\section{Sample}

Study participants were included if they met the following criteria: DSM-IV diagnosis of a mood, anxiety, or adjustment disorder; absence of a diagnosis of substance abuse or psychosis; absence of active suicidal ideation; agreement to remain on stable doses of any prescribed psychotropic medication for the duration of the study; and access to a computer, webcam, and the Internet. Eighteen participants met the study criteria.

\section{Procedures}

We invited eligible participants to choose the intervention format that they would feel most comfortable using for participation in group psychotherapy. Eight chose the on-line format and the remaining ten chose the face-to-face format. In both formats, the sessions were videoed and archived for subsequent analysis. Both interventions consisted of 13 weekly one-hour sessions of group CBT. The CBT group protocol was followed in both formats: the first 2 weeks of the intervention focused on socializing participants into the CBT model and developing group cohesion; weeks 3-4 centered on learning the basic behavioral interventions (eg, relaxation training and rating of mood); weeks 5-10 focused on cognitive and behavioral strategies for coping with stress, anxiety, and depression; and weeks 11-13 addressed termination of treatment, consolidation of gains, and training in relapse prevention. Both the on-line and face-to-face sessions were videoed and archived for subsequent analysis. The therapist was a trained and experienced CBT psychotherapist; however, supervision was provided by an expert CBT trainer to ensure reliable adherence to protocol in both formats. The clinical supervisor was the first author of this study, and is a $\mathrm{PhD}$ clinical psychologist with 10 years of professional experience ( 8 years heading a CBT service for mood and anxiety disorders at Baycrest, Toronto, Canada). 


\section{Technology platform}

Internet-based video conferencing group CBT was delivered using a password-protected web platform, ie, Caring for $\mathrm{Me}$ (CFMC) which was developed and evaluated in previous studies. ${ }^{15-17}$ The CFM website includes several features: an email component allowing participants to communicate with each other external to the group meetings; an asynchronous threaded discussion forum for exchanging thoughts; a video conferencing link for group meetings; and a resources link that provides an information handbook on depression and its diagnosis and treatment; CBT task documents uploaded for discussion during video conferencing treatment sessions; and relevant URLs for accessing reliable information about depression and its management. Website navigation is supported by a computer training manual distributed to all participants. All participants were provided with technology support as needed throughout the study by the project coordinator. During the sessions, all participants and therapist could see, hear, and interact with each other in real time. The participants appeared around the perimeter of the screen with the therapist in the center.

\section{Outcome measure}

The primary outcome measure was the BDI-II (Beck Depression Inventory Second Edition),${ }^{18}$ a validated instrument typically used for assessing the level of depression in outpatient treatment programs. The BDI-II was completed at baseline and at 3-month follow-up. BDI-II category cutoffs were as follows: 0-13, minimal depression; 14-19, mild depression; 20-28, moderate depression; and 29-63, severe depression. Higher total scores indicate more severe symptoms of depression.

\section{Data analysis}

The aim of the data analysis was four-fold: to examine reliable adherence to the CBT intervention protocol using each format; to examine differences, if any, in discussion themes by phase of therapy within each format; to examine withinsubject pre-post changes in score on the BDI-II; and describe participants' perceptions of using technology to access a health care service versus traditional in-clinic services.

\section{Treatment reliability and validity}

A standardized coding system rating levels of adherence to intervention strategies based on the CBT intervention manual was used, ${ }^{19}$ for example, adherence coding related to the standard CBT protocol, such as setting the agenda and reviewing and assigning homework. Following training and achievement of high interrater levels of agreement (80\%), two research assistants independently coded six sessions randomly selected from the pool of recorded sessions across each intervention group (face-to-face and video conferencing formats). Note that two groups were treated in each format, so six sessions were coded for each of four intervention groups.

\section{Analysis of group session themes}

While the themes discussed in CBT therapy were guided by the structure of the therapeutic protocol (cognitive-behavioral), it was important to extract the salient themes from each intervention format (Internet-based versus clinic-based). The methods described by Berg ${ }^{20}$ and Creswell ${ }^{21}$ were used to perform the qualitative analysis of group process themes in each delivery condition. Codes were developed, initial themes recorded, and researcher notes provided for careful documentation of the salient themes identified within and across groups. Two researchers independently engaged in this process using the transcriptions for each group to ensure accuracy of extracted themes.

\section{Results}

Eighteen subjects were included in the study (ten chose the face-to-face condition and eight the on-line condition). Seventy-two percent of the subjects were female, $100 \%$ were Caucasian, and their average age was 50.8 (range 39-64) years for the on-line group and 58.4 (range 40-71) years for the face-to-face group (see Table 1). On the basis of the DSM-IV diagnostic interview data, participants in each intervention format did not differ. Three participants withdrew (one in the face-to-face group and two in the video conferencing group) from the intervention program for reasons unrelated to the study intervention. The stated reasons for withdrawal were inability or unwillingness to make the time commitment for the intervention, scheduling difficulties, and, in one case, severity of symptoms, warranting referral for CBT on an individual basis (see Table 2).

With regard to the reliability and validity of treatment, interrater agreement on use of the CBT coding system was established at the $85 \%$ and $90 \%$ levels. Subsequently, two early, two middle, and two late sessions in each group (face-to-face and video conferencing) were coded and demonstrated that the CBT intervention was carried out reliably in both conditions.

Qualitative theme analysis was conducted on sessions 3, $4,7,8,12$, and 13 . The themes generated were similar between the two group intervention formats. The following key themes emerged from the analysis. 
Table I Patient demographics

\begin{tabular}{lll}
\hline & Face-to-face & Video conferencing \\
\hline Participants (n) & 10 & 8 \\
Mean subject age, years (range) & $58.4(40-71)$ & $50.8(39-64)$ \\
Sex (\% female) & 90 & 50 \\
Marital status (\% married) & 70 & 50 \\
Education (\% university/college or higher) & 80 & 62.5 \\
Employment status (\% employed) & 40 & 87.5 \\
\hline
\end{tabular}

Notes: Participants who withdrew from the study during various stages of intervention delivery were included in the calculations. Participants who were divorced, separated, or had a deceased spouse were considered as not married.

\section{Consistent struggle with the condition}

Participants discussed the challenge of understanding emotions, managing social interactions, developing personal coping methods, and focusing on pursuing strategies for change (eg, "What happens especially when I get real depressed, I just cut myself off from everybody and everything; it doesn't help my mood but I feel at those times I have zero energy and zero desire to do anything and I feel like I don't belong or fit").

\section{Low self-esteem and emotional triggers}

Participants engaged in group discussion about situations that exacerbated their negative self-perceptions. Common statements included, "I don't deem myself as valuable as others", "I don't know how to get along with people", and "I am not good enough".

\section{Relationships}

Stress experienced in relationships with family and friends was illustrated by the following statement: "When you end up doing more for somebody else, for me it turns into resentment, a feeling of being used".

\section{Managing work stress}

It was evident that the work environment was stressful, inducing anxiety for all members of the intervention groups (eg, “Come Friday I feel relieved that I don't have to come to work, I don't have to keep pretending I'm good and that

Table 2 Participants screened during recruitment

\begin{tabular}{lll}
\hline & Round I & Round 2 \\
\hline Participants (n) & 15 & 16 \\
Accepted & 10 & 10 \\
Percent acceptance & 66.7 & 62.5 \\
Withdrawals & 2 & 3 \\
Percent withdrawals & 20 & 30 \\
\hline
\end{tabular}

Notes: Participants who withdrew during various stages of intervention delivery were included in the acceptance criteria. Withdrawal indicates the number of participants accepted into the program but who chose to withdraw. everything is dandy and all that" and "What I struggle with on a daily basis is the anxiety once I'm at work").

According to the change in pre-post score on the BDI-II, similar proportions of participants in the face-to-face and video conference groups demonstrated positive, negative, or no change in severity of symptoms. Sixty percent of participants in each group showed improvement in their BDI-II score severity (eg, pre-post change from a moderate to low BDI-II score), with $20 \%$ moving to a more severe category and $20 \%$ showing no change in category (see Table 3 ).

\section{Analysis of 3-month follow-up data}

Despite the fact that all participants were group therapynaïve, participants in each group bonded and readily related to each other (eg, "When people spoke I could identify the same things in myself. It feels like we are not really different"). Group members responded positively to specific CBT intervention strategies (eg, "I found that this homework was really good because the one thing it did was it made me get moving"). The response of participants to using technology in a clinical context was positive overall (eg, 'I'm enjoying the group. I think that I'm slowly getting over the technology thing and it felt each week more like I was talking to other people rather than these images on a computer screen").

\section{Impact of technology: the therapist perspective}

The same CBT therapist delivered the intervention in both formats and found the first on-line group challenging in terms of implementing the CBT protocol and ensuring group cohesion. Technical glitches due to problems with the video conferencing software were frustrating for both the therapist and the study participants. For example, some subjects had difficulty logging on for a session, and the sound was occasionally disrupted during sessions. The study coordinator was always on-line during sessions to resolve any technical problems.

The therapist became more comfortable using the on-line CBT strategy after the first session, which was reflected 
Table 3 Change in BDI-II severity score

\begin{tabular}{llll}
\hline Face-to-face $(\mathbf{n}=\mathbf{1 0})$ & & Video $(\mathbf{n}=\mathbf{5})$ & Two moderate to low \\
\hline Improved & Two significant to low & Improved & One significant to moderate \\
Improved & Four moderate to low & Improved & One low to moderate \\
Worse & One low to moderate & Worse & One significant to significant \\
No change & Two low to low & No change & \\
No change & One significant to significant & & $60 \%$ showed positive change \\
& $60 \%$ showed positive change & & in BDI-II classification \\
\hline
\end{tabular}

Notes: For BDI-II pre-post change, similar proportions of participants in the face-to-face and video conferencing groups showed positive, negative, and no change in severity category. Sixty percent of participants in each group improved on their BDI-II score severity category (eg, pre-post change from moderate category to low BDI-II score category), $20 \%$ moved to a more severe category, and $20 \%$ showed no change. BDI-II category cutoffs were as follows: 0-13, minimal depression; I4-19, mild depression; 20-28, moderate depression; and 29-63, severe depression. Higher total scores indicate more severe depressive symptoms.

Abbreviation: BDI-II, Beck Depression Inventory Second Edition.

by her easier manner in structuring the CBT protocol and emphasizing key elements, such as specific follow-up with homework requests. The therapist described the experience of facilitating CBT in a technological milieu as a "learning curve", which was mastered by addressing the challenges of using technology and active clinical supervision.

\section{Discussion}

The main outcome of this study was that the group CBT intervention protocol was adhered to reliably in two formats, ie, face-to-face and on-line. Standards of practice were upheld for both conditions, and the discussion themes were similar between the two formats. Participants who completed the 13-week group CBT protocol had comparable pre-post BDI-II scores regardless of treatment format, ie, web video conferencing versus clinic-based face-to-face sessions. Sixty percent of participants in each group showed a positive change in classification of their BDI-II severity (eg, from moderate to low symptom levels). Our results are consistent with those of other studies of group CBT in older adults and, including one study which demonstrated improvement similar to patients included in a randomized controlled trial of group CBT for depression. ${ }^{22,23}$

In terms of the mood outcome measures, equivalent results across formats suggest several possibilities. First, fidelity to the CBT model was well established in this study. As such, the "active" ingredient of CBT was robust enough to negate the impact of any differences in the delivery format. In fact, a recent Cochrane review documented the effectiveness of therapist-delivered distance CBT (by phone, email, video conferencing, live chat, and print) for depression and anxiety in adults. ${ }^{24}$ Second, convenience factors associated with the video conferencing format, such as evening sessions and remote access to treatment in one's own home, may have compensated for the absence of in vivo interaction for some participants, especially for employed subjects who were unable to schedule treatment during working hours. In a review of therapeutic video conferencing for chronic physical and psychological disorders, Steel et al ${ }^{25}$ reported that the clinical outcomes of video conferencing were comparable with those found with in-person delivery. Video conferencing studies have shown a high level of patient satisfaction and a positive therapeutic alliance. Lastly, from both the therapist and participant perspectives, our results indicate that the video conferencing format did not compromise outcomes with regard to mood or thematic content of the sessions. Nonetheless, it must be acknowledged that freedom to choose the treatment modality may have impacted the results of this study. Participants may have felt more comfortable and adhered more to a personally chosen intervention format as opposed to an assigned one. Finally, the sample size of the study was small, and this is a clear study limitation. Our results are preliminary and need to be interpreted with caution until they are replicated in future studies.

In summary, our pilot study results suggest that group therapy delivered by Internet-based video conferencing may be suitable for treating depression and anxiety in adults. Offering CBT by remote access would constitute an achievement of both knowledge translation and clinical innovation. Internet-based video CBT group intervention is a client-centered, evidence-based, and cost-effective way to treat adults with mood and anxiety disorders, who may have difficulty accessing services in person due to health concerns, frailty, finances, location, or stigma. The limitations of this study are its small sample size and the fact that participants were able to choose their preferred treatment modality. Further studies with randomization of the treatment condition and a larger sample size are needed to validate the promising findings of the present study. 


\section{Acknowledgment}

This work was supported by an operating grant from the Canadian Institutes of Health Research (awarded to the second author).

\section{Disclosure}

The authors have no conflicts of interest to disclose with respect to any aspect of this research.

\section{References}

1. World Health Organization. Depression is a common illness and people suffering from depression need support and treatment. Geneva, Switzerland: World Health Organization; 2012. Available from: http://www.who.int/mediacentre/news/notes/2012/mental_health_ day_20121009/en/. Accessed March 19, 2014.

2. Blazer DG. Depression in late life: review and commentary. J Gerontol A Biol Sci Med Sci. 2003;58:249-265.

3. Schoevers RA, Deeg DJ, van Tilburg W, Beekman AT. Depression and generalized anxiety disorder: co-occurrence and longitudinal patterns in elderly patients. Am J Geriatr Psychiatry. 2005;13:31-39.

4. Wolitzky-Taylor KB, Castriotta N, Lenze EJ, Stanley MA, Craske MG. Anxiety disorders in older adults: a comprehensive review. Depress Anxiety. 2010;27:190-211.

5. Naughton C, Bennett K, Feely J. Prevalence of chronic disease in the elderly based on a national pharmacy claims database. Age Ageing. 2006; 35:633-636.

6. Katon WJ. Epidemiology and treatment of depression in patients with chronic medical illness. Dialogues in Clinical Neuroscience. 2011;13(1):7-23.

7. Beck AT, Weishaar ME. Cognitive therapy. In: Corsini RJ, Wedding D; editors. Current Psychotherapies. 6th ed. Itasca, IL, USA: Peacock; 2000.

8. Cappeliez P. Presentation of depression and response to group cognitive therapy with older adults. Journal of Clinical Geropsychology. 2000;6:165-174.

9. Cappeliez P, Latour D, Guirguis M. Relationship between certain characteristics of subjects and response to cognitive therapy for depressed older adults. Can J Aging. 1997;16:70-83.

10. Latour D, Cappeliez P. Pretherapy training for group cognitive therapy with depressed older adults. Can J Aging. 1994;13:221-235.

11. Bennet-Levy J, Richards D, Farrand P, et al; editors. Oxford Guide To Low Intensity CBT Interventions. London, UK: Oxford University Press; 2010.
12. Tucker M, Oie TPS. Is group more cost effective than individual cognitive behaviour therapy? The evidence is not solid yet. Behav Cogn Psychother. 2007;35:77-91.

13. Hawkley LC, Cacioppo JT. Loneliness and pathways to disease. Brain Behav Immun. 2003;17 Suppl 1:S98-S105.

14. Kaltenthaler E, Shackley P, Stevens K, Beverley C, Parry G, Chilcott J. Computerised cognitive behavioural therapy for depression and anxiety report commissioned by NHS R\&D HTA Programme on behalf of NICE. National Institute for Clinical Excellence; London, UK: 2002. Available from: http://admin.nice.org.uk/nicemedia/ live/11478/32491/32491.pdf. Accessed April 14, 2014.

15. Marziali E. The design and evaluation of e-health intervention programs for older adults. eHealth Int. 2008;4:6-13.

16. Marziali E, Damianakis T, Donahue P. Virtual support for family caregivers: theoretical framework, intervention model and outcome. J Technol Hum Serv. 2006;24:39-54.

17. Marziali E, Donahue P. Caring for others: Internet, video-conferencing group intervention for family caregivers of older adults with neurodegenerative disease. Gerontologist. 2006;46:398-403.

18. Beck AT, Steer RA, Brown GK. Manual for the Beck Depression Inventory-II. San Antonio, TX, USA: Psychological Corporation; 1996.

19. Thompson L, Gallagher-Thompson D, Dick LP. Cognitive-Behavioral Therapy for Late-Life Depression: A Therapist's Manual. Stanford, CA, USA: VA Palo Alto Health Care System and Stanford University; 1996.

20. Berg BL. Qualitative Research Methods for the Social Sciences. Boston, MA, USA: Allyn and Bacon; 1995.

21. Creswell JW. Qualitative Inquiry and Research Design: Choosing Among Five Traditions. Thousand Oaks, CA, USA: Sage Publications; 1998.

22. Krishna M, Jauhari A, Lepping P, Turner J, Crossley D, Krishnamoorthy A. Is group psychotherapy effective in older adults with depression? A systematic review. Int J Geriatr Psychiatry. 2011;26:331-340.

23. Turner A, Hambridge J, Baker A, Bowman J, McElduff P. Randomised controlled trial of group cognitive behaviour therapy versus brief intervention for depression in cardiac patients. Aust N ZJ Psychiatry. 2013; 47:235-243.

24. Olthuis JV, Watt MC, Stewart SH. Therapist-delivered distance cognitive behavioural therapy for anxiety disorders in adults protocol. Cochrane Database Syst Rev. 2011;3:CD009028. Available from: http://summaries.cochrane.org/CD009028/therapist-delivereddistance-cognitive-behavioural-therapy-for-anxiety-disorders-in-adults. Accessed March 20, 2014.

25. Steel K, Cox D, Garry H. Therapeutic videoconferencing interventions for the treatment of long-term conditions. J Telemed Telecare. 2011; 17:109-117.
Clinical Interventions in Aging

\section{Publish your work in this journal}

Clinical Interventions in Aging is an international, peer-reviewed journal focusing on evidence-based reports on the value or lack thereof of treatments intended to prevent or delay the onset of maladaptive correlates of aging in human beings. This journal is indexed on PubMed Central, MedLine,

\section{Dovepress}

CAS, Scopus and the Elsevier Bibliographic databases. The manuscript management system is completely online and includes a very quick and fair peer-review system, which is all easy to use. Visit http://www.dovepress. com/testimonials.php to read real quotes from published authors. 